\title{
Combined Cataract and Glaucoma Surgery: Trabeculectomy in Small Incision Cataract Surgery with Posterior Chamber Intra Ocular Lens (SISC with PCIOL) Implantation and its Success
}

\author{
A K M Shahidur Rahman Tarafder ${ }^{1}$, M Anwarul Kader ${ }^{2}$
}

\begin{abstract}
Objective
To asses the results are reducing and maintaining intraocular pressure of a patient having cataract with glaucoma, Performing Trabeculectomy (Filtration Surgery) and small incision cataract surgery (SICS) by the same wound \& same sitting.

Method and Materials

This study was done at Rajshahi Medical College Hospital from July 2006 to June 2008. Forty (40) patients of age ranging from 45 to 65 years were selected randomly having cataract with glaucoma. Intraocular pressures (IOP) were $14 \mathrm{mmHg}$ to $17 \mathrm{mmHg}$ preoperatively with antiglaucoma medication. Visual acuity of all patients was perception of light to counting finger (CF) half meter to one meter. Thirty eight (38) patients (95\%) had open angle glaucoma and two patients had chronic angle closure glaucoma. Informed consent was taken from all the patients. Trabeculectomy were done in the SICS wound. Side ports were made in every case. Delivery of nucleus, washing of cortical matter and introduction of PCIOL all are done under local anesthesia.

Result

The patients were followed up 2 weeks, 4 weeks, 4 months and 6 months interval. All patients had IOP within normal limit $(14-17 \mathrm{mmHg})$ with medication before surgery cataract of all patients were total, fundus were invisible due to cataract. In the first post operative day ( $1^{\text {st }}$ OPD) visual acuity of thirty eight (38) patients (95\%) were $6 / 24$ to $6 / 18$ and with pinhole $6 / 12$, except 2 (two) patients (5\%) among them 1 (one) (2.5\%) having hyphaema. Hyphaema were relieved after 3 days by using Tab. Anaroxyl 3 times daily for 2 days and vitamin ' $C$ ' (250mg) daily for 15 days. After 6 weeks all patients had IOP $11 \mathrm{mmHg}$ to $14 \mathrm{mmHg}$ without antiglaucoma medication and 39 (thirty nine) patients (95\%) had achieved corrected vision $6 / 12$ to $\% 6$. Except, 1 (one) (2.5\%) had vision lowering counting finger 5 meter due to end stage glaucoma, who had cup disc ratio (C:D.9) 5 (five) patients (12.5\%) developed polycystic bleb and rest diffuse functional bleb. Cup disc ration (C:D) of $39(95 \%)$ was 0.4 to 0.8 . no wound gap with iris proplase, thin bleb or fibrosed bleb with engorged overlying vessels were reported in any case.
\end{abstract}

Abstract

Conclusion

Trabeculectomy in the wound of SICS with PCIOL at the same sitting is effective both for management of glaucoma and cataract.

TAJ 2010; 23(1): 15-17

\section{Introduction}

Trabeculectomy is a filtration surgery. Various filtration surgeries have been tried since 1905 for glaucoma. But Trabeculectomy is the choice of operation for all types of glaucoma except infantile type. Trabeculectomy is still being done

${ }^{1}$ Associate Professor, Department of Ophthalmology, Rajshahi Medical College Hospital, Rajshahi.

${ }^{2}$ Assistant Professor, Department of Ophthalmology, Rajshahi Medical College Hospital, Rajshahi. 
throughout the world for last 20 years with some modifications and additions. Trabeculectomy lowers IOP creating a fistula which allows aqueous outflow from the anterior chamber to the sub tenon space. The fistula is protected or guarded by a superficial scleral flap ${ }^{1}$. There are some problems when trabeculectomy and cataract extraction done in a single setting of a patient having glaucoma with cataract. In SICS with PCIOL implantation the wound was made in the sclera 1.5-2mn from limbus. So Trabectomy can be done easily in the same wound and same sitting.

\section{Procedure}

Informed consent was taken from all the patient. Pupil was made dilated with Tropicamide (1\%) eye drop and phenylephrine eye drop 10\%. Fornix based conjunctival peritomy was done and Tenon capsule were separated from conjunctival at the area of operation of all cases. Cauterization of bleeding points were done through thermo cautary. Straight incision $6 \mathrm{~mm}$ to $7 \mathrm{~mm}$ were made 1.5 to $2 \mathrm{~mm}$ away from limbus by using keratome. Scleral tunnel were made and entry to anterior chamber (AC) were done. After introduction of viscoelastic substance into AC a side port was made and capsulorrhexis was done through it. The tunnel was enlarged in such a way so that externally it was $6 \mathrm{~mm}$ to $7 \mathrm{~mm} \&$ internally it was $10 \mathrm{~mm}$ to $11 \mathrm{~mm}$.Hydro dissection and hydrodelineation was done and nucleus was taken into AC. The nucleus was delivered by using irrigating vectis, washing of cortical mater was done and PCIOL was implanted. After introduction of viscoelastic substance (ocugel). The trabecular tissue were cut by side port knife, iridectomy was done by taking the iris through the inner lip of wound conjunctiva were made apposition by using 10-0 monofilament nylon. Visco elastic substance was removed by simco two way irrigation and aspiration canula. The function of trabeculectomy were checked by injecting ringers solution through the side port. Inj. Gentamycin and Dexamethason were given sub conjunctively. Eye was closed.

Tropical antibiotic, steroid were infiltered from first post operative day ( $1^{\text {st }}$ POD) upto 6 weeks and Tropicamide were infiltrated $1^{\text {st }}$ OPD up to 15 days. The patients were followed up 2 weeks, 4 weeks, 4 months and 6 months interval.

\section{Results}

Combined operation (Trabeculectomy SICS with PCIOL) were done in 40 eyes of 40 patient having glaucoma (IOP ranges from 29 to $37 \mathrm{mmHg}$ ) and dens cataract. All patients had IOP within normal limit 14-17 mmHg with premedication. Cataract of all patients were dens, fundus were not visible, after performing surgery (at $1^{\text {st }}$ POD) visual acuity of 38 patients (95\%) were $6 / 24$ to $6 / 18$ and with pinhole $6 / 18$ to $6 / 121$ (one) patient (2.5\%) having hyphaema and another one have C:D 0.9 (end stage glaucoma) and this 2 patients visual acuity were markedly reduced. AC reaction was mild to moderate. Hyphaema was reported in one case in the $1^{\text {st }}$ OPD and that was relieved after three days by using Tab. Anaroxyl 3 times daily for 3 days and Tab. Vitamin "C" (250mg) daily for 15 days. After 6 weeks all patients had IOP $10 \mathrm{mmHg}$ to $14 \mathrm{mmHg}$ without any anti-gluacoma therapy. Thirty nine patients (97.5\%) had achieved corrected vision $6 / 12$ to $6 / 6$. 1 patient (2.5\%) had visual acuity C.F. 5 meter due to end stage glaucoma (C:D.9). Five eyes (12.5) developed polycystic bleb and rest (87.5\%) diffuse functional bleb. Cup disc ratio (C:D) of 39 patients (97.5\%) were 0.4 to 0.8 . no wound gap, Irisprolapse thin bleb or fibrosed bleb with engorged overlying vessels was reported in any case.

\section{Discussion}

Both Contract and glaucoma are conditions that show increasing prevalence with aging. It is not surprising that many patients with glaucoma eventually developed contract either naturally or as a result of the effects of glaucoma therapy. The term glaucoma refers to a group of diseases that have a common characteristic optic neuropathy with associated visual field loss for which elevated intraocular pressure is one of the primary risk factor ${ }^{2}$. Glaucoma is not a curable disease but progression of optic neuropathy and associated visual field loss can be delayed by reducing IOP (primary risk factor) Even after maintaining the 
normal IOP long term follow up glaucoma cases have shown progressive loss of ganglion cell go on causing a characteristic loss of visual field but if IOP can be maintained in normal level survival of visual field is longer. There are many drugs available for lowering IOP and some are claiming for neuro protection but the drugs should be used regularly in proper time and proper doses. Many author have reported that early surgery is the answer of glaucoma management and it is true in developing countries like our because our patient particularly from rural area are very poor, Illiterate and have a tendency to not taking drugs regularly lack of follow up which is very important for medical management of glaucoma.

So when glaucoma patient need cataract surgery, then combined procedure cataract extraction and trabeculectomy in a single sitting is our choice of management in which we can avoid double operation in two different sittings which gives several benefits like cost-effectiveness and faster rehabilitation. ${ }^{3 .}$ Previously combined procedure was done in a single sitting by a special type of incision. But small incision cataract surgery (SICS) is a newer sutureless technique of cataract surgery becoming popular by its less post operative complication. Cost effectiveness and less time consuming surgery. Combined procedure in SICS is a challenge. In this study excision of tenons capsule were done, scleral tunnel were made and trabecletomy were done through same tunnel and found reduction of IOP 10mmHg of 17 $\mathrm{mmHg}$ in $97.5 \%$ cases after one year of surgery. Only one case developed raised IOP (23mmHg) after 4 months. He had severe post operative uveitis, out of 40 intraocular pressure were below $14 \mathrm{mmHg}$ in 35 patients (87.5\%) which is satisfactory result no doubt but we have to do this procedure and follow up to a huge number of patients to get a real picture.

\section{Conclusion}

Combined cataract and glaucoma surgery (extraction of lens and trabeculectomy) is the better treatment of a poor, illiterate patient of a rural area of our country where regular follow up facilities are not available and trabeculectomy in the wound of SICS may be the effective procedure of management of cataract and glaucoma both.

\section{References}

1. Jack J Kanski, Clinical Ophthalmology Elsevier Butter Worth Heinemann-Sixth Edition 2006 P. 430-431.

2. American Aeademy of Ophthalmology 2004-2005 section 10 p-1-231.

3. Gytun JL. Van Derkarr MA, Sanders V, Combined Cataract and glaucoma surgery, J cataract refract surg 199:20:121-1219. 